\title{
O Acesso das Pessoas Transexuais e Travestis à Atenção Primária à Saúde: uma revisão integrativa
}

\section{The Access of Transsexuals and Crossdressers to the Primary Health Care: an integrative review}

\section{El Acceso de las Personas Transexuales y Travestis a la Atención Primaria de Salud: una Revisión Integradora}

\author{
Lourenço Barros de Carvalho Pereira' ${ }^{1}$ Ana Cláudia Santos Chazan ${ }^{1}$ \\ ${ }^{1}$ Universidade do Estado do Rio de Janeiro. Rio de Janeiro, RJ, Brasil.
}

\section{Resumo}

Introdução: Travestis, transexuais e transgêneros (trans) fazem parte das minorias sexuais. São vítimas de estigmatização, discriminação e violência desde a infância, o que os leva muitas vezes à marginalização. Posto isso, se faz necessário políticas públicas que Ihes forneçam condições para o exercício da cidadania. O presente trabalho apresenta os resultados de uma revisão integrativa sobre o acesso das pessoas trans aos serviços de saúde da atenção básica. Métodos: Coleta de dados realizada em dezembro de 2017 nas plataformas MEDLINE, SciELO e LILACS, utilizando os descritores em associação: transexual, transgênero ou travesti, acesso e atenção primária, atenção básica ou saúde. Os estudos deveriam ser publicados a partir de 2007 e estarem disponíveis gratuitamente. Resultados: Dos 578 estudos encontrados (incluídos as repetições) foram selecionados 6, provenientes do Brasil, EUA e África do Sul. Cinco com metodologia qualitativa e uma revisão sistemática. O acesso à Atenção Primária à Saúde (APS) por parte da população trans encontra barreiras multidimensionais. Há entraves políticos, socioeconômicos, organizacionais, técnicos e simbólicos, porém as barreiras técnicas são as mais impactantes ao acesso. Conclusão: Para que haja um acesso equitativo, livre de preconceitos e de discriminação, e cuidado pautados na empatia e compaixão, faz-se necessário incluir a temática diversidade sexual e de gênero na formação dos profissionais de saúde na graduação, na pós-graduação e, principalmente, na educação permanente daqueles implicados aos cuidados das pessoas trans. A APS, ao fazer valer seus atributos, mostra-se como o cenário ideal para o cuidado longitudinal destas pessoas.

Palavras-chave: Acesso aos Serviços de Saúde; Atenção Primária à Saúde; Pessoas Transgênero; Medicina de Família e Comunidade

Como citar: Pereira LBC, Chazan ACS. O Acesso das Pessoas Transexuais e Travestis à Atenção Primária à Saúde: uma revisão integrativa. Rev Bras Med Fam Comunidade. 2019;14(41):1795. https://doi.org/10.5712/rbmfc14(41)1795
Autor correspondente: Lourenço Barros de Carvalho Pereira. E-mail: loubcp@gmail.com

Fonte de financiamento: declaram não haver.

Parecer CEP: não se aplica.

Procedência e revisão por pares: revisado por pares. Recebido em: 30/06/2018. Aprovado em: 02/03/2019. 


\begin{abstract}
Introduction: Crossdressers, transsexuals and transgenders (trans) are part of the sexual minorities. They have been victims of stigmatization, discrimination and violence since childhood, which often leads to marginalization. Considering this, it is necessary to have public policies that provide them the conditions for the exercise of citizenship. The present paper presents the results of an integrative review on the access of trans people to primary health care (PHC). Methods: Data collection performed in December 2017 on the MEDLINE, SciELO and LILACS platforms, using the descriptors in association: transsexual, transgender or transvestite, access and primary care, basic care or health. The studies should be published as of 2007 and available for free. Results: Of the 578 studies found (including replicates), 06 were selected from Brazil, USA and South Africa. Five of them used qualitative methodology and one is a systematic review. Access to $\mathrm{PHC}$ by trans people encounters multidimensional barriers. There are political, socioeconomic, organizational, technical and symbolic obstacles, but the technical barriers are the most impacting to access. Conclusion: In order to ensure equal access, free of prejudice and discrimination, and care based on empathy and compassion, it is necessary to include the theme of sexual and gender diversity in the training of health professionals in undergraduate, postgraduate and, in the permanent education of those involved in the care of transgender people. PHC, when asserting its attributes, shows itself as the ideal setting for the longitudinal care of these people.
\end{abstract}

Keywords: Health Services Accessibility; Primary Health Care; Transgender Persons; Family Practice

\title{
Resumen
}

Introducción: Travestis, transexuales y transgéneros (trans) hacen parte de las minorías sexuales. Son víctimas de estigmatización, discriminación y violencia desde la infancia, lo que los lleva a la marginación. Por eso, se hacen necesarias políticas públicas que les proporcionen condiciones para el ejercicio de la ciudadanía. El presente trabajo presenta los resultados de una revisión integrativa sobre el acceso de las personas a los servicios de salud de la atención básica (AB). Métodos: Recolección de datos para la revisión efectuada en diciembre de 2017 en las plataformas MEDLINE, SciELO y LILACS, utilizando los descriptores en asociación: transexual, transgénero o travesti, acceso y atención primaria, atención básica o salud. Los estudios deberían publicarse a partir de 2007 y estar disponibles gratuitamente. Resultados: De los 578 estudios encontrados (incluidas las repeticiones) fueron seleccionados 06. Los artículos provenían de Brasil, EE.UU. y Sudáfrica. Cinco de ellos usaron metodología cualitativa, siendo una revisión sistemática. El acceso a la $A B$ por parte de la población trans encuentra diversas barreras, siendo multidimensionales. Hay obstáculos políticos, socioeconómicos, organizacionales, técnicos y simbólicos, pero las barreras técnicas son las más impactantes. Conclusión: Para que haya un acceso ecuánime, libre de prejuicios y de discriminación, y cuidado pautados en la empatía y compasión del paciente, se hace necesario incluir la temática diversidad sexual y de género en la formación de los profesionales de salud en la graduación, en el postgrado y, principalmente, en la educación permanente de aquellos implicados en el cuidado de las personas trans. La APS, al hacer valer sus atributos, se muestra como el escenario ideal para el cuidado longitudinal de estas personas.

Palabras clave: Accesibilidad a los Servicios de Salud; Atención Primaria de Salud; Personas Transgénero; Medicina Familiar y Comunitaria

\section{Introdução}

Travestis, transexuais e transgêneros, neste texto chamados em coletivo por trans, ainda representam uma parcela com menor visibilidade dentro do movimento LGBT (lésbicas, gays, bissexuais, transexuais e travestis) no Brasil e no mundo. Em relação à saúde, essas pessoas possuem especificidades que as diferenciam neste coletivo.

O Brasil é o país que mais mata transexuais e travestis no mundo: entre janeiro de 2008 e setembro de 2017 foram mais de 1071 homicídios, ${ }^{1}$ tendo sido registrados 182 assassinatos de transexuais somente de outubro de 2016 a setembro de $2017 .{ }^{1}$

Transfobia é o nome que se dá à repulsa e/ou preconceito contra pessoas trans, ${ }^{2}$ gerando a discriminação e sofrimento nos diferentes momentos e espaços de suas vidas: na família, no ambiente escolar, nos ambientes sociais e no trabalho. A exclusão social causada pela transfobia é evidenciada pela realidade de menor nível de escolaridade, subemprego e prostituição a que estão sujeitas estas pessoas, principalmente em grandes cidades. ${ }^{3}$ As travestis ainda sofrem mais da estigmatização que as outras pessoas do coletivo LGBT por serem determinadas a modificar o corpo e manterem-se na ambiguidade entre o feminino e o masculino. ${ }^{4}$ 
Estigma, neste cenário, pode ser considerado como o aspecto negativo, o status inferior e a relativa impotência que a sociedade atribui coletivamente a qualquer comportamento, identidade, relacionamento ou comunidade não heteronormativa (ou não cisnormativa). ${ }^{5-7}$

Na constituição brasileira de 1988 sob o artigo 194 está posto que "cabe ao Estado garantir a seguridade social", ${ }^{8}$ entendendo a seguridade social como acesso à saúde, previdência e assistência social. No mesmo texto, o artigo 195 regulamenta as ações e serviços públicos em saúde e cria o Sistema Único de Saúde (SUS), que tem como diretrizes a universalidade do acesso, a equidade e a integralidade na assistência. ${ }^{9}$ Em adequação a essas diretrizes, o Estado vem ao longo desses anos implementando diferentes políticas públicas voltadas para grupos que necessitam de atenção especial, dada a sua vulnerabilidade social, dentre eles, o grupo trans.

A Figura 1 resume os marcos históricos das conquistas trans dos últimos anos.

\section{Transexualidades, Travestilidades e atenção primária à saúde}

Ainda que os tratamentos de reafirmação de gênero, segundo alguns autores tenham sido historicamente negligenciados pelo SUS, ${ }^{10}$ existe no âmbito da saúde, e também nos movimentos sociais, uma construção de ideias de políticas públicas de assistência à população trans que vão além do diagnóstico e cirurgia de transgenitalização, que envolve um cuidado integral e que vai ao encontro dos anseios por autonomia, empoderamento e exercício pleno da cidadania. ${ }^{3,11}$

AAtenção Básica ( $A B)$ ou Atenção Primária a Saúde (APS) - termos que neste artigo são utilizados como sinônimos - é a base organizadora e a porta de entrada prioritária dentro da rede de atenção à saúde (RAS) no país, cenário ideal para o cuidado continuado, longitudinal e coordenado dos indivíduos. ${ }^{11}$ Então, falar de travestilidade e transexualidade na perspectiva da APS é tornar visíveis questões que há pouco tempo eram desconhecidas pela população geral.

Dado que a APS possui uma lógica de trabalho que valoriza o cuidado centrado nas pessoas e não nas doenças, e tem no vínculo a possibilidade de concretizar a continuidade, a integralidade e a coordenação deste cuidado, será que a população trans se sente acolhida nas unidades da APS?

O objetivo deste artigo é apresentar os resultados de uma revisão integrativa sobre o acesso das pessoas trans aos serviços de saúde da atenção básica. Acesso esse, entendido como o grau de facilidade com que as pessoas obtêm cuidados em saúde. ${ }^{12}$

\section{Métodos}

A revisão integrativa foi adotada como método de agrupamento dos dados coletados sobre o tema proposto. Trata-se de uma ampla abordagem metodológica que permite a inclusão de estudos experimentais e não experimentais, dados da literatura teórica e empírica sobre aquilo que se deseja analisar. Por meio dela, é possível gerar um panorama consistente e compreensível de conceitos complexos, teorias ou problemas de saúde relevantes ao médico de família e comunidade. ${ }^{13}$ Assim como os outros métodos de revisão, a revisão integrativa está atrelada à Prática Baseada em Evidências (PBE).

Os dados foram buscados a fim de responder às perguntas norteadoras: Como se dá o acesso das pessoas trans ao sistema de saúde através da APS? Há barreiras e desafios a serem superados? 
$2^{\circ}$ oferta do curso UNA-SUS/UERJ Política Nacional LGBT

- $\quad 3^{a}$ Conferência Nacional de Políticas Públicas e Direitos Humanos de LGBT

campos: identidade de gênero e orientação sexual

- $\quad 1^{\text {a }}$ oferta do curso UNA-SUS/UERJ Política Nacional LGBT

- Lançamento do livro Transexualidade e Travestilidade na saúde/MS 2014 Seminário sobre Travestilidade e Transexualidade no SUS

Reforça-se criminalização da Igbtfobia.

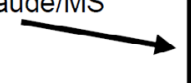

Grupos reivindicam maior cobertura do processo transexualizador (PrTr) pelo SUS

2013 Inclusão do nome social no cartão do SUS

- Ampliação do PrTr

- $\quad 2^{a}$ Conferência Nacional de Políticas Públicas e Direitos Humanos de LGBT

Política reconhece que a discriminação por identidade de gênero e orientação sexual incide na determinação social do processo saúde-adoecimento das pessoas LGBT expostas

2009 Aprovada a Política Nacional de Saúde LGBT no CNS

Promover a equidade no acesso ao SUS ao implementar a Política Nacional de Saúde LGBT

$$
\text { PrTr implementado no SUS }
$$

- $\quad 1^{\text {a }}$ Conferência Nacional de Políticas Públicas e Direitos Humanos de LGBT discussão dos problemas que envolvem os direitos humanos e políticas públicas de LGBT

2007

$13^{a}$ Conferência Nacional de Saúde

2006 Conquistas pelos militantes LGBT no CNS

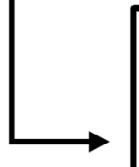

Garantir o acesso de LGBT e outras populações vulneráveis aos serviços do SUS, e para isso se faria uma política

2004 Criação do Comitê Técnico LGBT/MS

- Brasil Sem Homofobia

- 29 de janeiro é criado o Dia da Visibilidade Trans nacional.

1990

ASTRAL: Associação de travestis e liberados do Rio de Janeiro

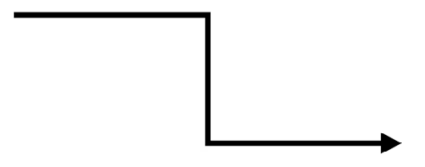

Primeira organização de travestis a discutir questões de cidadania e promover articulação nacional de pessoas trans.

Figura 1. Marcos históricos das conquistas trans nos últimos anos.

Adaptação de figura do artigo: A Política Nacional de Saúde Integral de Lésbicas, Gays, Bissexuais e Transgêneros (LGBT) e o acesso ao Processo Transexualizador no Sistema Único de Saúde (SUS): avanços e desafios. ${ }^{11}$ (2017) 
A revisão deu-se conforme os seis passos propostos por Ganong: ${ }^{14}$ formulação do problema, busca de dados, avaliação dos dados coletados, análise crítica e interpretação dos estudos incluídos, apresentação, e discussão dos resultados.

Foi feita pesquisa nas bases de dados LILACS (Literatura Latino-Americana e do Caribe em Ciências da Saúde), SciELO (Scientific Electronic Library Online) e MEDLINE (Medical Literature Analysis and Retrievel System Online), em dezembro de 2017. Foram usados os descritores "transgênero", "travesti" ou "transexual”; "acesso" e "atenção primária", "atenção básica" ou "saúde" e seus equivalentes em inglês.

As publicações deveriam estar disponíveis na íntegra e gratuitamente, estar em inglês, português, francês ou espanhol. Foram selecionados apenas artigos dos últimos dez anos, posto que em 2007, no âmbito da $13^{a}$ Conferência Nacional de Saúde, foi definido pela primeira vez que era obrigação das três esferas de governo garantir o acesso aos serviços e bens de saúde pela população LGBT. ${ }^{11}$ A exclusão dos trabalhos se deu pela leitura dos resumos e observação do conteúdo em relação a pergunta norteadora.

\section{Da avaliação e processo de análise}

Para a extração de dados do material coletado, foi usado instrumento adaptado pelos autores, a partir do validado por Ursi. ${ }^{13} \mathrm{O}$ título do artigo, periódico, país, idioma, ano da publicação, tipo de estudo, objetivo, avaliação do rigor metodológico e intervenções, implicações e propostas são algumas informações que o instrumento exige.

A fim de avaliar a qualidade da publicação, foi aplicado o CASP Checklist (Critical Appraisal Skills Programme $)^{15}$ apropriado para cada tipo de estudo.

Para avaliar a qualidade do nível de evidências, os artigos foram classificados por meio da hierarquia das evidências, segundo o delineamento da pesquisa, como proposto por Dawson: ${ }^{16}$

Nível 1: evidências resultantes da meta-análise de múltiplos estudos clínicos controlados e randomizados;

Nível 2: evidências obtidas em estudos individuais com delineamento experimental;

Nível 3: evidências de estudos quase-experimentais;

Nível 4: evidências de estudos descritivos (não experimentais) ou com abordagem qualitativa;

Nível 5: evidências provenientes de relatos de caso ou de experiência;

Nível 6: evidências baseadas em opiniões de especialistas.

\section{Resultados}

Na base de dados LILACS, foram encontrados 14 artigos relacionados a transgênero, 3 artigos a travesti e 6 artigos a transexual. Na base de dados MEDLINE em referência a transgênero se encontram 392 artigos, 144 a transexual e 8 a travestis. Ao passo que, na base SciELO, 7 artigos foram encontrados com o termo transgênero, 3 com transexual e apenas 1 com travesti.

Se fossem escolhidos somente os estudos que se limitavam às pessoas travestis, transexuais ou transgêneros, não haveria um número adequado de artigos para analisar, por isso, foram aceitos artigos que também tratassem da temática LGBT, desde que contemplassem a pergunta norteadora. Após análise dos artigos baseados nos critérios de inclusão e exclusão, foram pré-selecionados então 2 artigos na base 
de dados LILACS, 3 artigos na base de dados MEDLINE e um artigo na base de dados SciELO. Após a avaliação de qualidade com a ferramenta citada acima, todos os seis artigos foram considerados com qualidade satisfatória. Observa-se então que, em sua grande maioria, os estudos encontrados não tratam das temáticas acesso e atenção primária concomitantemente.

Os artigos selecionados foram provenientes da África do Sul (1), ${ }^{17}$ Brasil (3) ${ }^{18-20}$ e Estados Unidos (2). ${ }^{21,22}$ Cinco dos seis artigos foram publicados em revistas relacionadas ao tema saúde e 1 foi publicado em um periódico da área da administração. ${ }^{18}$ Quatro são estudos descritivos e/ou qualitativos, 1 revisão sistemática ${ }^{17}$ e 1 baseado em relato de caso. ${ }^{21}$ Quanto ao idioma, 3 deles em inglês, 3 em português. 0 Quadro 1 mostra um resumo dos 6 artigos em questão.

Quadro 1. Análise dos artigos selecionados, quanto ao delineamento, nível de evidência e objetivo ou questão de investigação.

\begin{tabular}{|c|c|c|c|}
\hline Título & Delineamento & Nível de evidência & Objetivo ou questão de investigação \\
\hline $\begin{array}{l}\text { Desigualdades no SUS: o caso } \\
\text { de atendimento às travestis é } \\
\text { "babado" }\end{array}$ & Pesquisa qualitativa & 4 & $\begin{array}{l}\text { Analisar a percepção, sentimentos e dificuldades } \\
\text { enfrentadas pelas travestis para terem acesso aos } \\
\text { serviços de saúde e serem atendidas de forma igualitária } \\
\text { aos demais segmentos da população. Pretende também } \\
\text { indicar maneiras de melhorar a assistência, diminuir } \\
\text { vulnerabilidades, aproximar os profissionais de saúde } \\
\text { do grupo estudado e levar ao público, através de sua } \\
\text { publicação, os resultados encontrados }\end{array}$ \\
\hline $\begin{array}{l}\text { How Should Physicians } \\
\text { Refer When Referral Options } \\
\text { Are Limited for Transgender } \\
\text { Patients? }\end{array}$ & $\begin{array}{l}\text { Ensaio baseado em } \\
\text { um caso clínico }\end{array}$ & 5 & $\begin{array}{l}\text { Argumentar que uma rotina de redução de danos } \\
\text { e consentimento informado, com forte ênfase na } \\
\text { continuidade dos cuidados em um ambiente de atenção } \\
\text { primária, deve orientar questões sobre como referenciar } \\
\text { pacientes transgêneros para profissionais e instituições } \\
\text { de cuidados em saúde a partir da atenção primária }\end{array}$ \\
\hline $\begin{array}{l}\text { Injustice to transsexual } \\
\text { women in a hetero-normative } \\
\text { healthcare system }\end{array}$ & Revisão sistemática & 4 & $\begin{array}{l}\text { Explorar os problemas de saúde que as mulheres } \\
\text { transexuais experimentam, e fazer uma descrição do } \\
\text { sistema de saúde hetero-normativo na África do Sul, após } \\
\text { isso, são feitas recomendações apropriadas para prática } \\
\text { e pesquisa. }\end{array}$ \\
\hline $\begin{array}{l}\text { O que esperam pessoas trans } \\
\text { do Sistema Único de Saúde? }\end{array}$ & Estudo qualitativo & 4 & $\begin{array}{l}\text { Apresentar as propostas, soluções e reivindicações } \\
\text { apresentadas por pessoas trans aos seus atendimentos } \\
\text { nos serviços de saúde no âmbito do Sistema Único de } \\
\text { Saúde (SUS). }\end{array}$ \\
\hline $\begin{array}{l}\text { A clínica em movimento na } \\
\text { saúde de TTTS: caminho } \\
\text { para materialização do SUS } \\
\text { entre travestis, transsexuais e } \\
\text { transgêneros }\end{array}$ & Ensaio & 4 & $\begin{array}{l}\text { Discutir a problemática envolvendo a saúde do "universo } \\
\text { TTT" (travestis, transexuais e transgêneros) ressaltando } \\
\text { o limitado direito à saúde e ao acesso a ações e serviços } \\
\text { de saúde, o preconceito e a discriminação sofridos } \\
\text { pela inadequação dos profissionais e dos recursos } \\
\text { tecnológicos utilizados }\end{array}$ \\
\hline $\begin{array}{l}\text { Outness, Stigma, and Primary } \\
\text { Health Care Utilization among } \\
\text { Rural LGBT Populations }\end{array}$ & $\begin{array}{l}\text { Estudo } \\
\text { quali-quantitativo }\end{array}$ & 4 & $\begin{array}{l}\text { O objetivo deste estudo é determinar se níveis mais } \\
\text { elevados de estigma e/ou níveis mais baixos de } \\
\text { autoafirmação se correlacionam com o menor acesso } \\
\text { aos cuidados de saúde primários para populações LGBT } \\
\text { rurais. Como nossa amostra contém um amplo espectro } \\
\text { de orientações sexuais e identidades de gênero, também } \\
\text { tentaremos destacar as diferenças de estigma, discrição } \\
\text { e acesso aos cuidados entre esses grupos. }\end{array}$ \\
\hline
\end{tabular}


Com relação à amostra, três não necessitavam de plano amostral por se tratarem ou de artigo de revisão ou por ser baseado em relato de experiência. ${ }^{17,20,21}$ Os demais estudos explicitaram os critérios para escolha da amostra, 2 por conveniência, 1 de forma aleatória, sendo que esse discorreu sobre os possíveis vieses com relação à sua amostra. ${ }^{22}$

Três desses artigos tinham por objetivo analisar as percepções das pessoas trans e LGBT em relação ao acesso e atendimento nos sistemas de saúde, ${ }^{18,19,22}$ um defendia uma mudança na abordagem a essas pessoas na APS ${ }^{21}$ e os demais eram revisões da literatura com o objetivo de analisar as barreiras e problemas enfrentados pelas pessoas trans no acesso aos serviços de saúde. ${ }^{17,20}$ O Quadro 2 apresenta de forma sucinta os achados relacionados ao acesso e suas barreiras e desafios. O Quadro 3 contém propostas feitas para a prática e formação médicas e das demais profissões da saúde para contribuir de forma direta ou indireta com a superação dessas barreiras e desafios.

\section{Discussão}

Os resultados encontrados ratificam a importância do tema.

Ainda que tenha sido encontrada uma quantidade considerável de estudos voltados para a população trans nos últimos 10 anos, quando adicionado o termo de busca "atenção primária", poucos trabalhos foram identificados, o que indica que ainda há muito a se investir na produção de conhecimento, principalmente no Brasil, onde a APS se coloca com coordenadora da RAS e porta de entrada do SUS. ${ }^{16,23,24}$

A maioria dos artigos envolvendo a população trans tem como objeto a cirurgia de redesignação sexual ou a vulnerabilidade ao HIVISIDA, limitando os conceitos de "acesso" e "saúde" da população trans somente a esses aspectos, negligenciando o cuidado integral preconizado pelas diretrizes do SUS e políticas públicas de cuidados a população trans.

\section{Acesso à APS/AB}

O acesso é uma importante categoria a ser considerada na formulação e implementação de políticas públicas, devido ao seu potencial de melhoria para a organização do SUS. ${ }^{25}$

Entre os fatores limitantes para o acesso destacam-se a baixa acessibilidade, sistema fragmentado, focalizado e não integral, ausência de equidade e acolhimento, ausência de descentralização e regionalização da atenção. ${ }^{25}$ Por acessibilidade se entende a distância e localização entre serviços de saúde e os usuários, as barreiras geográficas, tempo para o deslocamento e custos implicados, bem como a oferta de serviço e profissionais qualificados para a atenção desejada. ${ }^{26-28}$

As desigualdades de acesso são um dos principais problemas enfrentados para que a APS e o SUS funcionem efetivamente.

No caso da APS, podemos considerar que ele é o atributo, sem o qual os outros ficam impossibilitados de acontecer. Para que o acesso equitativo na APS seja uma realidade, usuários, trabalhadores e gestores devem colaborar nos colegiados gestores das unidades, para identificar e solucionar as barreiras, ${ }^{27}$ que tornam imperceptíveis as demandas e necessidades em saúde da população trans. 
Quadro 2. Panorama do acesso, barreiras e desafios.

\begin{tabular}{|c|c|}
\hline$O$ acesso atualmente & Barreiras e desafios \\
\hline $\begin{array}{l}\text { - Passam por situações constrangedoras e muitas vezes tem } \\
\text { acesso negado. }{ }^{18}\end{array}$ & $\begin{array}{l}\text { - Muitas vezes o médico não toca no corpo da travesti, isso pode } \\
\text { enfraquecer o vínculo e causar afastamento do serviço. }{ }^{18}\end{array}$ \\
\hline - Impossibilidade de poder escolher onde ser atendida. ${ }^{18}$ & $\begin{array}{l}\text { - Travestis relatam medo de serem maltratadas nos serviços de } \\
\text { saúde, o que favorece a não utilização. }{ }^{18}\end{array}$ \\
\hline $\begin{array}{l}\text { - O acolhimento em locais onde há mais profissionais LGBT } \\
\text { aparenta ser mais adequado. }{ }^{18}\end{array}$ & $\begin{array}{l}\text { - A não observação do nome social causa um grande desconforto } \\
\text { nas pessoas, e também na travesti. }{ }^{18}\end{array}$ \\
\hline $\begin{array}{l}\text { - Pode haver automedicação devido medo de constrangimentos } \\
\text { no acolhimento. }{ }^{18}\end{array}$ & $\begin{array}{l}\text { - É comum por parte dos profissionais de saúde associarem a } \\
\text { imagem da travesti à infecção por HIV. Assim, todo ou qualquer } \\
\text { sintoma sempre remete a possibilidade de infecção podendo } \\
\text { causar iatrogenia ou atrasar um diagnóstico. }{ }^{18}\end{array}$ \\
\hline $\begin{array}{l}\text { - Trans fingem não escutar quando são chamadas pelo nome } \\
\text { masculino da identidade, nos serviços de saúde, por vergonha, } \\
\text { contudo, de modo geral elas acabam se expondo. }{ }^{18}\end{array}$ & $\begin{array}{l}\text { - Os profissionais tendem a acreditar que qualquer sintoma seja } \\
\text { associado a um efeito colateral da hormonização. }{ }^{21}\end{array}$ \\
\hline $\begin{array}{l}\text { - A violência simbólica que sofrem no sistema de saúde nem } \\
\text { sempre é percebida como tal. }{ }^{18}\end{array}$ & $\begin{array}{l}\text { - Em geral, os postos de saúde (unidades de AB) frequentados } \\
\text { por pessoas trans têm conceito baixo quanto ao acolhimento e } \\
\text { serviços prestados. }^{18}\end{array}$ \\
\hline $\begin{array}{l}\text { - Nos EUA, tanto afrodescendentes quanto transgêneros } \\
\text { imigrantes sem plano de saúde e pobres têm acesso mínimo } \\
\text { aos serviços de saúde. }{ }^{17}\end{array}$ & $\begin{array}{l}\text { - Pessoas trans relatam preconceito no acolhimento e mau } \\
\text { atendimento por parte dos profissionais. A capacidade dos } \\
\text { profissionais de saúde em lidar com temas que envolvam a } \\
\text { transexualidade é vital para o acesso e uso apropriados dos } \\
\text { serviços de saúde. Nos EUA } 101 \text { mulheres trans indicaram isso } \\
\text { como a principal barreira de acesso. }{ }^{17}\end{array}$ \\
\hline $\begin{array}{l}\text { - Qualquer sexualidade diferente da heterossexualidade é } \\
\text { considerada ilegal na maioria significativa dos países africanos } \\
\text { e a África possui uma cultura heteronormativa muito forte, } \\
\text { dificultando o acesso. }{ }^{17}\end{array}$ & $\begin{array}{l}\text { - Para população trans, altos níveis de estigma, nas suas três } \\
\text { formas (por antecipação, internalizados e públicos), predizem } \\
\text { resultado baixo no escore de saúde. }{ }^{22}\end{array}$ \\
\hline $\begin{array}{l}\text { - Na América do Norte e do Sul o acesso aos serviços disponíveis } \\
\text { para a comunidade LGBT permanece em grande parte limitado, } \\
\text { ou inexistem serviços de saúde disponíveis para a comunidade } \\
\text { transexual. }{ }^{17}\end{array}$ & $\begin{array}{l}\text { - Pessoas trans que relatam sofrerem mais por estigma acessam } \\
\text { menos os serviços de saúde que cisgêneros. } .^{22}\end{array}$ \\
\hline $\begin{array}{l}\text { - Transgêneros têm três vezes mais chances de escolher um } \\
\text { "profissional que já atende outros LGBT" (está entre seus três } \\
\text { principais motivos de escolha), além de que escolhe muito mais } \\
\text { o "profissional com conhecimento específico LGBT" do que } \\
\text { cisgêneros. }{ }^{22}\end{array}$ & $\begin{array}{l}\text { - O fato de se assumir como trans para o profissional de saúde } \\
\text { não garante escore de saúde alto, o que pode indicar falta de } \\
\text { conhecimento dos médicos em recomendações de saúde para } \\
\text { essas pessoas. }{ }^{22}\end{array}$ \\
\hline \multirow[t]{2}{*}{$\begin{array}{l}\text { - Em centros especializados de atenção LGBT, as travestis e os } \\
\text { profissionais se sentem mais à vontade com seus corpos, há } \\
\text { então, maior probabilidade de haver exame físico. }{ }^{18}\end{array}$} & $\begin{array}{l}\text { - Pessoas trans têm a probabilidade aproximadamente três vezes } \\
\text { maior que pessoas cis de viajar mais de uma hora para uma } \\
\text { consulta de atenção primária. } 10 \% \text { delas frequentam clínicas } \\
\text { específicas para LGBTs. }{ }^{22}\end{array}$ \\
\hline & $\begin{array}{l}\text { - Pesquisa com transexuais de Massachusetts verificou } \\
\text { que a discriminação estava associada a } 24 \% \text { dos pacientes } \\
\text { transgêneros adiando o cuidado rotineiro ou preventivo e } 11 \% \\
\text { adiando cuidados que mais tarde resultaram em tratamento de } \\
\text { emergência. }{ }^{22}\end{array}$ \\
\hline
\end{tabular}

Segundo Assis e Jesus, o acesso aos serviços de saúde é multidimensional envolvendo aspectos políticos, econômicos, sociais, organizativos, técnicos e simbólicos. ${ }^{25}$ Observou-se neste estudo que em relação as pessoas trans há barreiras ao acesso em todas as dimensões.

Houve muitos avanços na dimensão política, tanto no nível nacional, com a criação das políticas afirmativas e de equidade, a partir da participação social do segmento trans no Conselho Nacional de Saúde (CNS), quanto em nível estadual, como no caso de São Paulo com a Portaria CCD/CRT no a-1, de 
Quadro 3. Propostas e intervenções no âmbito da formação e da prática em saúde.

\begin{tabular}{|c|c|}
\hline Propostas para a prática em saúde & Proposta para a formação em saúde \\
\hline $\begin{array}{l}\text { - Troca do modelo atual, patologizante de acesso ao processo } \\
\text { transexualizador (PrTr), para o modelo de consentimento-livre e } \\
\text { esclarecido. }^{21}\end{array}$ & $\begin{array}{l}\text { - Capacitação dos profissionais da APS com relação ao cuidado } \\
\text { de pessoas trans para que se possa minimizar os danos físicos e } \\
\text { emocionais e criar um ambiente adequado, aumentando assim o } \\
\text { conforto do paciente em se assumir e reforçando relacionamento } \\
\text { médico-paciente. }{ }^{19,21}\end{array}$ \\
\hline $\begin{array}{l}\text { - Troca do modelo de cuidado médico-centrado para aquele } \\
\text { centrado na pessoa. }{ }^{21}\end{array}$ & $\begin{array}{l}\text { - Necessita-se de mais pesquisa com pessoas trans no mundo, } \\
\text { em especial no meio médico. Incluindo os temas de saúde geral, } \\
\text { morbimortalidade, violência transfóbica e estupro. }{ }^{17}\end{array}$ \\
\hline $\begin{array}{l}\text { - Cuidado continuado na atenção primária, para além do PrTr. } \\
\text { A APS tem como atributos o cuidado longitudinal e integral das } \\
\text { pessoas. }{ }^{21}\end{array}$ & $\begin{array}{l}\text { - Transfobia deve ser tratada separadamente das outras formas } \\
\text { de violência para que haja medidas corretivas e campanhas de } \\
\text { prevenção voltadas especificamente para esse tema. }{ }^{17}\end{array}$ \\
\hline $\begin{array}{l}\text { - Descentralização e capilarização das instituições que oferecem } \\
\text { PrTr para que seja disponível perto das pessoas. }{ }^{19,21}\end{array}$ & $\begin{array}{l}\text { - Incorporar conteúdo obrigatório sobre educação sexual integral } \\
\text { nas diferentes carreiras da saúde desde a graduação. }{ }^{18}\end{array}$ \\
\hline $\begin{array}{l}\text { - Melhor aconselhamento médico sobre as hormonizações e } \\
\text { disponibilização dos hormônios para profissionais do sexo. }{ }^{19}\end{array}$ & $\begin{array}{l}\text { - Desenvolver treinamento institucional sobre o conteúdo das } \\
\text { portarias e políticas voltadas para a população trans. }{ }^{19}\end{array}$ \\
\hline $\begin{array}{l}\text { - Necessidade de ampliar a disponibilidade das próteses } \\
\text { mamárias para evitar o uso de silicone industrial por parte das } \\
\text { mulheres trans e travestis. }{ }^{19}\end{array}$ & - Educação permanente sobre a temática. ${ }^{17}$ \\
\hline \multicolumn{2}{|l|}{$\begin{array}{l}\text { - A observação e uso do nome social em todas as instituições de } \\
\text { saúde. }{ }^{19}\end{array}$} \\
\hline \multicolumn{2}{|l|}{ - Humanização do acolhimento às pessoas trans. ${ }^{19}$} \\
\hline \multicolumn{2}{|l|}{$\begin{array}{l}\text { - Dia exclusivo para atendimento de pessoas trans ou ambulatório } \\
\text { específico para elas. }{ }^{19}\end{array}$} \\
\hline \multicolumn{2}{|l|}{$\begin{array}{l}\text { - Presença de funcionários trans principalmente ACS, devido sua } \\
\text { proximidade com os usuários. }{ }^{19}\end{array}$} \\
\hline \multicolumn{2}{|l|}{$\begin{array}{l}\text { - Hormonização gerida pelo clínico da APS. Tendo em conta a } \\
\text { competência cultural dos clínicos que possam fornecer cuidados } \\
\text { de afirmação de gênero e a importância da continuidade do } \\
\text { cuidado, não é só aceitável, mas também, preferível que um } \\
\text { médico da APS faça a gestão hormonal. }{ }^{21}\end{array}$} \\
\hline $\begin{array}{l}\text { - O profissional da APS que encaminha para atenção } \\
\text { especializada deve avaliar tanto a capacidade técnica do } \\
\text { profissional que irá atender quanto também sua vontade e } \\
\text { capacidade de desenvolver planos de cuidados que refletem } \\
\text { os desejos e necessidades do paciente. } .^{21}\end{array}$ & \\
\hline
\end{tabular}

27 de janeiro de $2010,{ }^{28}$ que criou um protocolo clínico para os ambulatórios de saúde trans; e em nível municipal, exemplo de Florianópolis com o Ambulatório de Atenção Primária à Saúde para Pessoas Trans (AAPST). ${ }^{11}$

$\mathrm{Na}$ dimensão econômico-social observa-se que o cuidado ainda é centralizado em hospitais em grandes cidades, havendo necessidade de aumentar a descentralização e regionalização do atendimento à população trans, principalmente em áreas rurais. Algo que pode e deve ser efetivado pela atuação da APS. A distribuição territorial da APS diminui os custos com deslocamento para o usuário e está culturalmente mais próxima dele. Também é preciso que as três esferas de poder garantam os investimentos necessários, de acordo com cada nível de complexidade de atenção. Vale ressaltar que, em muitas localidades, os municípios não entendem o cuidado à população trans como de competência municipal, ${ }^{29}$ já que as portarias vigentes visam a organização dos serviços de média e alta complexidade, fato que pode contribuir com menos investimentos na APS. 
O que temos hoje no Brasil é ainda uma RAS desintegrada, o que, do ponto de vista da organização do acesso, é um fator muito importante para a sua ineficiência. ${ }^{5,11}$ Além disso, a APS deve funcionar como porta de entrada, e deve fazê-lo bem, para tal é preciso qualidade técnica.

A dimensão técnica do acesso envolve acolhimento, integralidade do cuidado, vínculo, compromisso e qualidade assistencial. ${ }^{25}$ Essa dimensão foi apontada em todos os estudos como a mais impactante no acesso e satisfação com os serviços de saúde.

Observou-se que nesta dimensão a barreira se expressa pelo não acolhimento e pelo atendimento inadequado, por profissional não capacitado e pela falta de competência na integralidade do cuidado, ${ }^{18-21}$ agravado pelo modelo de atenção centralizado nos hospitais, sem contrarreferência para a APS. ${ }^{25}$

A não observação do nome social, a incapacidade em manejar questões próprias do universo LGBT, somado ao modelo médico hegemônico, paternalista, que não compartilha o projeto terapêutico, que prioriza o seu saber científico em detrimento da necessidade de saúde das trans, são exemplos de ações que levam ao constrangimento e insegurança das pessoas trans quando procuram os serviços de saúde. ${ }^{18,21}$

O fato das travestis saírem de suas consultas "deprimidas" ou "para baixo"18,21 revela que não há um acolhimento adequado pelos profissionais de saúde, o que contraria o princípio de igualdade do SUS. ${ }^{18}$ Os danos emocionais prejudicam a confiança na relação médico-paciente, afastando-os do serviço. ${ }^{19,30}$ É de suma importância que o médico seja formado para trabalhar com a lógica de redução de danos emocionais, não somente dos físicos.

Hoje o que se observa é que os serviços de saúde se apresentam como locais de manutenção das desigualdades e preconceitos da sociedade, em vez de serem locais de acolhimento e proteção. ${ }^{18,20,31}$ A própria linguagem médica, muitas vezes técnica e incompreensível, ainda que não seja a intenção, mostra-se como uma barreira, uma forma de manter o distanciamento social, algo que é resultado e reforço do cuidado centrado no médico e não no usuário. ${ }^{18}$

Foi constatado também que as barreiras simbólicas, aquelas que envolvem a subjetividade, a cultura e crenças das pessoas, ainda estão muito presentes no acesso à APS e ao PrTr.

Parker e Aggleton ${ }^{32}$ afirmam que os profissionais de saúde se mantêm distantes dos direitos humanos e acreditam que isso é uma competência do Estado, não entendendo o seu papel como "representantes do estado" ao acolher dentro do SUS, pessoas discriminadas e estigmatizadas socialmente. ${ }^{18}$

O estigma sexual e de gênero também pode explicar a baixa utilização da APS. O medo de serem maltratadas e as inúmeras situações constrangedoras, ou de negação do acesso, são realidades que afastam essas pessoas trans dos serviços de saúde. Elas infelizmente continuam a receber um tratamento diferenciado, como se fossem cidadãos com menos direitos do que os demais. ${ }^{20}$

A heteronormatividade é uma forma de discriminação presente nas instituições, mas ainda não é aceita como tal. ${ }^{17}$ A dicotomia de cuidado presente nos programas governamentais de saúde do homem e saúde da mulher não permite a introdução daquelas pessoas que não sem encaixam dentro destas "caixas". ${ }^{17}$ 


\section{A APS como cenário ideal para o cuidado}

Os achados revelam que a transição, seja ela hormonal ou cirúrgica, é somente uma das necessidades em saúde das pessoas trans, ${ }^{10,33}$ que clamam por acolhimento e atendimento livre de discriminação e cuidado integral. $\mathrm{O}$ atendimento médico, muitas vezes marcado por preconceitos e focado no risco de infecção por HIV e efeitos colaterais do PrTr, acaba por afugentar as pessoas trans dos serviços de saúde, levando a automedicação e a diminuição da autopercepção de saúde-doença. ${ }^{22,31}$

Muitas vezes, o motivo da consulta é uma questão comum, que independe do status de transexual, transgênero ou travesti.

A "síndrome do braço quebrado trans" (trans broken arm syndrome) é uma expressão que tem sido utilizada para descrever o fato dos "profissionais de saúde assumirem que todas as questões médicas apresentadas por uma pessoa trans se devem ao fato da pessoa ser trans". ${ }^{21}$ As consequências desse preconceito são a não identificação e resolução do problema, a criação de novas demandas, intervenções médicas desnecessárias e muitas vezes a supressão do tratamento transexualizador. ${ }^{10,34}$

Espera-se que o profissional da APS, e principalmente o médico de família conheça melhor seus pacientes do que os especialistas focais e sejam familiarizados com os diversos aspectos da saúde, família e bem-estar de sua população. Assim, se capacitados, eles podem e devem prescrever hormônios para seus pacientes e acompanhá-los, pois estão bem equipados para entender os efeitos da terapia - incluindo quais sintomas não estão relacionados a ela. ${ }^{35}$ Essa continuidade e integralidade do cuidado são partes inerentes da rotina da APS. ${ }^{24,33}$

Mas para que isso ocorra efetivamente, é mais do que necessário que o assunto seja abordado na graduação e na pós-graduação, ${ }^{31}$ principalmente nos programas de residência e especialização em saúde da família.

A hormonização é uma competência clínica, e como qualquer outra pode ser desenvolvida pelos médicos de família e comunidade. O manejo laboratorial da transição hormonal é tão complexo quanto outros manejos laboratoriais feitos na APS. ${ }^{19}$ Assim, usando a óptica da patologização, as transidentidades merecem ser cuidadas na APS assim como outras pessoas portadoras de afecções comuns e crônicas.

Esse cuidado voltado para doenças crônicas é do escopo da APS e seu manejo não difere do de pessoas que não fazem hormonioterapia. A diferença aqui é que pessoas usando hormônios tem um fator de risco a mais para desenvolver doenças cardiovasculares, ${ }^{17}$ e outras complicações inerentes à cronicidade desse processo.

A clínica peripatética, como sugerido por Freire et al. ${ }^{20}$ dialoga com a realidade da APS, como proposta de abordagem à população trans. Estes autores reforçam a competência cultural e territorialidade presentes na APS e na ESF, indicando que as ações devem ocorrer nos territórios geográfico e existencial dos sujeitos. Também relacionam a importância de envolver as subjetividades da pessoa no cuidado integral, algo que no modelo biomédico vigente, não acontece, ou acontece de forma segmentada. 
A APS além de ter de enfrentar as questões de acesso (barreiras em suas dimensões), que obrigatoriamente antecedem a coordenação do cuidado, tem problemas e desafios grandes no que tange a implementação de uma rede que permita a integração entre os níveis de atenção. ${ }^{16}$ Então, após as dificuldades para adentrar o SUS, as pessoas trans ainda encontram dificuldades para se locomover dentro da rede, apesar de que já houve muito avanço na fluidez dessas pessoas no SUS. ${ }^{10}$

\section{Necessidades e propostas trans}

Os profissionais de saúde devem estar aptos e capacitados para cuidar das pessoas trans. Para que isso seja uma realidade, e para que todo serviço de saúde possa: garantir acesso, trabalhar na lógica de redução de risco físico e emocional, atuar na eliminação do preconceito e da discriminação da população LGBT, garantir o uso do nome social e inclusão de temáticas relacionadas com estes grupos nas práticas cotidianas, precisamos pôr em prática algumas mudanças.

A primeira delas se refere à formação profissional em saúde. A diversidade de gênero e sexual deve ser abordada tanto na graduação quanto nas especializações médicas e de outras carreiras da saúde. Fazse necessário que o profissional médico esteja acostumado com o tema, e que saiba manejar os problemas dessa população de forma integral, lembrando da importância das subjetividades, do entendimento da discriminação e da estigmatização no processo de saúde adoecimento das pessoas.

A prática diária também deve ser modificada. O profissional não deve assumir a orientação sexual ou identidade de gênero considerando a aparência ou outras características da pessoa. Perguntar sobre os hábitos sexuais de forma aberta, cordial e não heteronormativa deve ser rotineiro ao médico. Perguntas simples, como por exemplo: "Como você deseja ser chamado(a)?" ou "Como você se identifica e gostaria que fosse identificado?" podem ser suficientes para reforçar o vínculo médico-paciente e transformar o ambiente num local mais acolhedor e confortável para a pessoa trans. ${ }^{35}$

Os estigmas internalizados são considerados como grandes propulsores de baixa autoestima. De acordo com Whitehead et al., ${ }^{22} 65 \%$ das pessoas trans de áreas rurais nos EUA tem sintomas relacionados a depressão, um número 15\% maior que a população cisgênero do mesmo lugar. Questionar o paciente sobre, e ajudá-lo a transpor essas barreiras é um dever do médico. Julgamentos e comentários moralistas apenas servem para aumentar a discriminação e a estigmatização destas pessoas. Transexuais, transgêneros e travestis apreciam quando os profissionais tomam ciência de suas limitações e de seus preconceitos. ${ }^{36}$

Alguns estudos sugerem que a queda de andrógenos, como resultado das hormonização para alcançar um fenótipo feminino, pode levar à depressão. ${ }^{17}$ Porém, devemos levar em consideração se a influência da discriminação, da estigmatização e consequente marginalização (intensificados à medida que os caracteres feminino e masculino se misturam mais) não são mais importantes para o descompasso da saúde mental das pessoas trans.

Mulheres transexuais podem estar potencialmente mais propensas ao suicídio devido sua marginalização. Por isso, a transfobia deve ser tratada separadamente das outras formas de violência para que haja medidas corretivas e campanhas de prevenção voltadas especificamente para esse tema, pois ela é a mais letal das formas de lgbtfobia. ${ }^{17}$ 
Além disso, a identidade de gênero só deve ser levada em consideração caso seja imprescindível naquele momento.

Para entender a importância que as modificações corporais têm para as trans, é necessário compreender que "é através do corpo que elas se fazem sujeito". ${ }^{37}$

Uma vez que a transformação do corpo por meio de hormônios pode ser suficiente para garantir a elas um sentido de identidade, nem sempre as cirurgias de transgenitalização são necessárias. Não é o órgão sexual o que mais importa, mas sim o exercício da cidadania, ${ }^{20}$ que começa com a adequação do nome.

Ainda no que tange às modificações corporais, há, no Brasil inclusive, e em outras partes do mundo a dependência de laudo psiquiátrico "liberando" a pessoa a fazer os procedimentos cirúrgicos, observando-se o medo, por parte de médicos, de um "arrependimento" no pós-operatório transexualizador. ${ }^{31}$

Nesse ponto cabe apontar duas questões. A primeira diz respeito à autonomia do sujeito, a quem cabe decidir sobre as modificações em seu corpo. Conforme proposto por Dietz e Halem, ${ }^{21}$ o sujeito deve ter sua autonomia preservada e deve ser ele a decidir sobre os procedimentos que deseja, e não o médico. A postura paternalista - o médico é quem escolhe o que o paciente deve ou não fazer - deve ser ultrapassada e o cuidado deve ser centrado na pessoa e em suas necessidades. A abordagem usando o consentimento informado (livre e esclarecido) é a preconizada pelos guias internacionais pois através dela o cuidado é iniciado pelo paciente e sua autonomia é incentivada. ${ }^{21}$

A segunda questão é sobre a seletividade desse tipo de comportamento médico. Quantos resultados de procedimentos estéticos cirúrgicos (mamoplastias, rinoplastias, entre outros) não atendem às expectativas dos pacientes e nem por isso é solicitado laudo psiquiátrico para sua efetivação? Essa seletividade é pautada no preconceito, na desconfiança e na patologização da pessoa trans por parte do médico. ${ }^{31}$

No ano de 2013 entrou em tramitação o projeto de lei (PL) “João Nery” Lei da identidade de Gênero ${ }^{38}$ - em homenagem ao primeiro transexual masculino do Brasil a fazer cirurgia de redesignação sexual. Esse $\mathrm{PL}$, caso aprovado, garante o reconhecimento legal da identidade de gênero das pessoas transexuais, amplia o atendimento nas redes de saúde, inclusive na rede privada e suplementar por meio dos planos de saúde, que hoje não possuem cobertura para esse tipo de atendimento.

Interessante notar que essa lei não prevê que pessoas transexuais se submetam ao PrTr ou à cirurgia de redesignação para terem acesso à troca de nome e gênero em seus documentos oficiais. Além disso, diminui para 18 anos a idade mínima para se submeter aos procedimentos cirúrgicos e simplifica o acesso, abolindo o diagnóstico de disforia de gênero e o laudo psiquiátrico. Para realizar os procedimentos, então, vai bastar apenas o consentimento livre e esclarecido, mudança que, como vimos, segue as tendências internacionais.

O médico de família e comunidade tem em seus princípios o domínio do método clínico centrado na pessoa, o conhecimento dos problemas de saúde mais comuns e os menos frequentes. Deve ser capaz de transitar pelos territórios, se adaptando às demandas locais e, assim, como recurso de sua população advogar pela comunidade, a fim de identificar as desigualdades e defender um acesso e atenção mais equitativos. ${ }^{5}$ 
O acesso da população LGBT e particularmente das pessoas trans à APS pode ser garantido seguindo esses princípios. Formar profissionais alinhados a isso é dever dos programas de residência médica.

\section{A APS potencializadora de ações intersetoriais}

As adversidades presentes na vida das pessoas transexuais, transgênero e travestis começam muitas vezes na infância. Nesse período começam a se sentir diferentes, e logo já enfrentam a discriminação e estigmatização. $O$ analfabetismo ou a não finalização dos estudos costumam ser comuns nesse contexto. ${ }^{3}$

É também na infância que surgem as primeiras ideias do transformar-se, de mostrar-se como deseja ser, ${ }^{39}$ que vão amadurecer ao longo do tempo e ser um propulsor para a luta pela cidadania. ${ }^{3}$ Porém, a vontade de criar uma identidade própria tem um preço social a ser pago. ${ }^{18}$

Desfechos negativos podem ser evitados promovendo-se melhorias na dimensão simbólica do acesso através do trabalho sobre apoio familiar, suporte social e a estabilidade emocional dos indivíduos na APS. Intervenções desde a infância, com o indivíduo e sua família podem ser benéficos. Essas condições são conhecidas como motores de resiliência para enfrentar os preconceitos e discriminações a que estão sujeitos. Para tal, o desenvolvimento técnico dos profissionais se faz necessário.

Como foi evidenciado, para que as pessoas trans tenham uma melhor qualidade de vida não basta somente ações no âmbito da saúde. O processo de saúde-adoecimento é um resultado de fatores determinantes em todos os aspectos da vida. Por isso, princípios de cidadania e respeito às diversidades sexuais e de gênero devem ser debatidos em seu aspecto micro e macropolíticos e devem ser pautadas no cotidiano das pessoas e problematizada na educação básica. ${ }^{11}$ AAPS pode ser um grande espaço para promover ações intersetoriais e promoção das causas LGBT.

\section{Limitações}

O presente estudo é resultado de pesquisa bibliográfica em algumas bases de dados que não dão acesso à literatura cinzenta. Desta forma, podem ter escapado elementos importantes para ampliar a discussão. Além disso, a escolha dos artigos por meio da leitura dos resumos pode ter levado a vieses relacionados à seleção.

\section{Conclusões}

As desigualdades de acesso encontram-se como um dos principais problemas a serem enfrentados para que o SUS possa funcionar conforme seus princípios e diretrizes. E para que o acesso na APS seja equitativo, garantindo a entrada de pessoas trans faz-se necessário ampliar o debate sobre diversidade sexual e de gênero para espaços onde ainda é muito desconfortável falar sobre. ${ }^{18}$

Políticas de saúde devem ser capazes de eliminar as barreiras geográfica, econômica e cultural entre os serviços e a comunidade, além de garantir que as pessoas trans permaneçam sob o cuidado integral de profissionais de saúde.

Essa temática deve estar presente na formação em saúde desde a graduação e reforçada nas residências e especializações. É preciso que os médicos sejam habilidosos no acolhimento e que o cuidado seja centrado na pessoa que está diante deles em um evento marcado por estigmas e medos. ${ }^{18}$ 
É indispensável que se continue rompendo a discriminação e a consequente violência institucionalizada; que se promova o respeito à singularidade dos sujeitos e o combate a todas as formas de normatização que impliquem na exclusão e discriminação dessas pessoas.

A prática da medicina de família e comunidade deve ser inclusiva. Promover o respeito à cooperação é um dever deste especialista e sua educação permanente deve ser orientada pelas necessidades de saúde da população sob o seu cuidado.

\section{Contribuição dos autores}

Concepção e/ou delineamento do estudo: LBCP, ACC. Aquisição, análise ou interpretação dos dados: LBCP. Redação preliminar: LBCP. Revisão crítica da versão preliminar: ACC.

Todos os autores aprovaram a versão final e concordaram com prestar contas sobre todos os aspectos do trabalho.

\section{Conflito de interesses}

Declaram não haver.

\section{Referências}

1. TvT Research Project. Trans Murder Monitoring. Transrespect versus Transphobia Worldwide (TvT) project website; 2016 [acesso 2017 Set 8]. Disponível em: www.transrespect.org/en/research/trans-murder-monitoring/

2. Priberam. Priberam Informática; 2017 [acesso 2017 Set 8]. Disponível em: http://www.priberam.pt/

3. Romano VF. As Travestis no Programa Saúde da Família da Lapa. Saúde Soc. 2008;17(2):211-9.

4. Benendetti MR. Toda feita: o corpo e o gênero das travestis. Rio de Janeiro: Garamond; 2005.

5. Abreu de Jesus WLA, Assis MMA. Revisão sistemática sobre o conceito de acesso nos serviços de saúde: contribuições do planejamento. Ciênc Saúde Coletiva. 2010;15(1):161-70. https://doi.org/10.1590/S1413-81232010000100022

6. Broeiro P. Justiça social e lei dos cuidados inversos. Rev Port Med Geral Fam. 2016;32(3):167-9.

7. Giovanella L, Fleury S. Universalidade da Atenção à Saúde: acesso como categoria de análise. In: Eibenschutz C, org. Política de Saúde: o público e o privado. Rio de Janeiro: Fiocruz; 1995. p. 177-98.

8. Brasil. Presidência da República/Casa Civil/Subchefia para assuntos jurídicos. Constituição da República Federativa do Brasil de 1988. Brasília: Presidência da República; 1988.

9. Brasil. Presidência da República. Lei 8080/90 I Lei no 8.080, de 19 de setembro de 1990. Dispõe sobre as condições para a promoção, proteção e recuperação da saúde, a organização e o funcionamento dos serviços correspondentes e dá outras providências. Brasília: Presidência da República; 1990.

10. Brasil. Ministério da Saúde. Secretaria de Gestão Estratégica e Participativa. Departamento de Apoio à Gestão Participativa. Transexualidade e Travestilidade na Saúde. Brasília: Ministério da Saúde; 2015. 194 p.

11. Popadiuk GS, Oliveira DC, Signorelli MC. A Política Nacional de Saúde Integral de Lésbicas, Gays, Bissexuais e Transgêneros (LGBT) e o acesso ao Processo Transexualizador no Sistema Único de Saúde (SUS): avanços e desafios. Ciênc Saúde Coletiva.2017;22(5):1509-20. https://doi.org/10.1590/1413-81232017225.32782016

12. Travassos C, Martins M. Uma revisão sobre os conceitos de acesso e utilização de serviços de saúde. Cad Saúde Pública. 2004;20(Suppl 2):S190-S198.

13. Souza MT, Silva MD, Carvalho R. Revisão integrativa: o que é e como fazer. Einstein. 2010;8(1 Pt 1):102-6.

14. Ganong LH. Integrative reviews of nursing research. Res Nurs Health. 1987;10(1):1-11. https://doi.org/10.1002/nur.4770100103

15. Critical Appraisal Skills Programme (CASP) [acesso 2017 Dez 28]. Disponível em: www.casp-uk.net/casp-tools-checklists 
16. Rodrigues LBB, Silva PCS, Peruhype RC, Palha PF, Popolin MC, Crispim JA, et al. A atenção primária à saúde na coordenação das redes de atenção: uma revisão integrativa. Ciênc Saúde Coletiva. 2014;19(2):343-52. https://doi.org/10.1590/1413-81232014192.18032012

17. Newman-Valentine D, Duma S. Injustice to transsexual women in a hetero-normative healthcare system. Afr J Prim Health Care Fam Med. 2014;6(1):a574. https://doi.org/10.4102/phcfm.v6i1.574

18. Muller MI, Knauth DR. Desigualdades no SUS: o caso de atendimento às travestis é 'babado'! Cad EBAPE.BR. 2008;6(2):1-14.

19. Rocon PC, Sodré F, Zamboni J, Rodrigues A, Roseiro MCFB. O que esperam pessoas trans do Sistema Único de Saúde? Interface (Botucatu). 2018;22(64):43-53.

20. Freire EC, Araujo FCA, Souza AC, Marques D. A clínica em movimento na saúde de TTTS: caminho para materialização do SUS entre travestis, transexuais e transgêneros. Saúde Debate. 2013;37(98):477-84. https://doi.org/10.1590/S0103-11042013000300011

21. Dietz E, Halem J. How Should Physicians Refer When Referral Options Are Limited for Transgender Patients? AMA J Ethics. 2016;18(11):1070-8.

22. Whitehead J, Shaver J, Stephenson R. Outness, Stigma, and Primary Health Care Utilization among Rural LGBT Populations. PLoS One. 2016;11(1):e0146139. https://doi.org/10.1371/journal.pone.0146139

23. Brasil. Ministério da Saúde. Secretaria de Gestão Estratégica e Participativa. Departamento de Apoio à Gestão Participativa. Política Nacional de Saúde Integral de Lésbicas, Gays, Bissexuais, Travestis e Transexuais. Brasília: Ministério da Saúde; 2013.

24. Starfield B. Atenção Primária: Equilíbrio Entre Necessidades de Saúde, Serviços e Tecnologia. Brasília: UNESCO, Ministério da Saúde; 2002.

25. Assis MMA, Jesus WLA. Acesso aos serviços de saúde: abordagens, conceitos, políticas e modelos de análise. Ciênc Saúde Coletiva. 2012;17(11):2865-75. https://doi.org/10.1590/S1413-81232012001100002

26. Unglert CVS. O enfoque da acessibilidade no planejamento da localização e dimensão de serviços de saúde. Rev Saúde Pública. 1990;24(6):445-52. https://doi.org/10.1590/S0034-89101990000600002

27. Silva NN, Pedroso GC, Puccini RF, Furlani WJ. Desigualdades sociais e uso de serviços de saúde: evidências de análise estratificada. Rev Saúde Pública. 2000;34(1):44-9. https://doi.org/10.1590/S0034-89102000000100009

28. Coordenadoria de Controle de Doenças, Centro de Referência e Treinamento - Dst/Aids (SP/BR). Portaria CCD/CRT- nº a-1, de 27 de janeiro de 2010. Cria o protocolo clínico nos ambulatórios de saúde para travestis e transexuais. Diário Oficial do Estado; Poder Executivo, São Paulo, SP, 28 jan. 2010. Seção I. São Paulo: Diário Oficial. p. 33.

29. Arán M, Murta D, Lionço T. Transexualidade e Saúde Pública no Brasil. Ciênc Saúde Coletiva. 2009;14(4):1141-9.

30. Silva SM, Spiassi AL, Alves DC, Guedes DJ, Leigo RO. Redução de danos: estratégia de cuidado com populações vulneráveis na cidade de Santo André - SP. Saúde Soc. 2009;18(Suppl 2):100-3.

31. Neer AF. Obstáculos y facilitadores para garantizar el derecho a la salud integral trans en el Gran Buenos Aires y La Plata. Rev Argent Salud Pública. 2016;7(29):26-30.

32. Parker R, Aggleton P, orgs. Cidadania e Direitos, № 1: Estigma, Discriminação e AIDS. Rio de Janeiro: Associação Brasileira Interdisciplinar de AIDS - ABIA; 2001

33. Reisner SL, Bradford J, Hopwood R, Gonzalez A, Makadon H, Todisco D, et al. Comprehensive transgender healthcare: the gender affirming clinical and public health model of Fenway Health. J Urban Health. 2015;92(3):584-92. https://doi.org/10.1007/s11524-0159947-2

34. Brasil. Ministério da Saúde. Carta dos direitos dos usuários da saúde. $2^{\text {a }}$ ed. Brasília: Ministério da Saúde; 2007.9 p.

35. Deutsch MB, ed.; Center of Excellence for Transgender Health. Guidelines for the Primary and Gender-Affirming Care of Transgender and Gender Nonbinary People. 2nd ed. San Francisco; University of California; 2016.

36. Lewis EB, Vincent B, Bret A, Gibson S, Walsh RJ. What Your Patient Is Thinking - I am your trans patient. BMJ. 2017;357:j2963. https://doi.org/10.1136/bmj.j2963

37. Pennington J, Knight T. Through the lens of hetero-normative assumptions: re-thinking attitudes towards gay parenting. Cult Health Sex. 2011;13(1):59-72. http://dx.doi.org/10.1080/13691058.2010.519049

38. Projeto de Lei 5002/2013. Lei João Nery, Lei da identidade de Gênero. Dispõe sobre o direito à identidade de gênero e altera o artigo 58 da Lei 6.015 de 1973. Brasília: Câmara dos Deputados; 2013.

39. Nery JW. Viagem Solitária: memórias de um transexual 30 anos depois. São Paulo: LeYa Brasil; 2011. 